\title{
Questes
}

Revue pluridisciplinaire d'études médiévales

\section{Grivoiserie, pornographie, scatologie : conclusion}

\section{Marie Dupuy et Mathilde Grodet}

\section{Q OpenEdition}

\section{Journals}

\section{Édition électronique}

URL : http://journals.openedition.org/questes/2681

DOI : 10.4000/questes.2681

ISSN : 2109-9472

\section{Éditeur}

Les Amis de Questes

\section{Édition imprimée}

Date de publication : 15 juin 2011

Pagination : 123-127

ISSN : 2102-7188

\section{Référence électronique}

Marie Dupuy et Mathilde Grodet, « Grivoiserie, pornographie, scatologie : conclusion », Questes [En ligne], 21 | 2011, mis en ligne le 15 janvier 2014, consulté le 15 septembre 2020. URL : http:// journals.openedition.org/questes/2681 


\section{Conclusion}

\section{Marie DUPUY et Mathilde GRODET}

Le thème proposé pour ce bulletin abordait trois notions: la grivoiserie, la pornographie et la scatologie. Au moment d'apporter une conclusion aux divers questionnements qui ouvraient la réflexion, force est de constater que les différentes contributions semblent exclure la pornographie du champ littéraire pour la période médiévale. Le mot n’est d'ailleurs jamais utilisé dans les articles, en raison sans doute de la valeur artistique des œuvres étudiées. La notion elle-même n’est pourtant pas totalement absente de l'univers médiéval et transparaît çà et là dans les textes, à travers le vocabulaire employé ou les descriptions très explicites, mais surtout à travers la dimension vénale de la sexualité telle qu'elle apparaît par exemple dans le fabliau du Sohait des vez que cite Caroline Foscallo, où une femme rêve qu'elle se rend sur un « marché aux vits ». Ce sont toutefois la grivoiserie et la scatologie qui ont été le plus largement évoquées au fil de ces analyses, toutes deux incluses dans le champ plus vaste de l'obscène qu'elles ont contribué à cerner. La grivoiserie en particulier offre, grâce au rire, une façon simple d'aborder sans détour la sexualité dans les textes du Moyen Âge. Contrairement à ce que nous aurions pu attendre, la scatologie, quant à elle, est présente dans tous les genres littéraires: dans les genres comiques évoqués par Francesco Montorsi, mais aussi de façon plus surprenante dans les genres dits nobles, comme la chanson de geste, mise en question par Chloé Chalumeau, ou la lyrique des troubadours, étudiée par Pierre Levron. Grivoiserie et scatologie peuvent également être liées. Marie-Emmanuelle Simon fait remarquer que le glissement de l'une à l'autre s'effectue dans les moralités à partir de la notion de péché de chair prise au sens large. Du plaisir de 
bouche au plaisir sexuel, on parvient finalement à la déjection. Pierre Levron signale aussi un intéressant cas d'ondinisme où plaisir sexuel et plaisir scatologique sont mêlés.

À la lumière de ces études, un vaste champ d'interprétation se dessine. Les fonctions comique et ludique, pour commencer, occupent une place de choix dans les finalités possibles de ces textes. Elles prennent leur source dans le sexe joyeux ou la scatologie ridicule des fabliaux et des farces notamment. Se met alors en place une langue propre au grivois, faite d'exagérations, d'hyperboles et de répétitions. La représentation se fait sur un mode littéral, omettant le plus souvent la métaphore pour donner à voir l'obscène à l'aide du verbe cru. Caroline Foscallo nous montre par ailleurs que les fabliaux se prêtent particulièrement au quiproquo, à la manipulation du langage et au double sens. Les œuvres dramatiques, quant à elles, permettent de démultiplier les jeux grivois dans l'écho visuel toujours possible qu'offrent les mises en scène de textes licencieux; l'absence de sources irréfutables laisse cependant ces dernières à l'imagination des Modernes, comme le fait remarquer Marie-Emmanuelle Simon. Mais dire le sexe et l'ordure se fait paradoxalement au moyen d'un vocabulaire assez limité, voire répétitif et stéréotypé : un rire libérateur accompagne la transgression des tabous verbaux, répétés à l'envi et reconnus comme tels par les auditeurs. Chloé Chalumeau nous invite à voir qu'à travers ces exemples, c'est plus généralement le rapport au langage qui doit être interrogé. Si le plaisir des textes repose en partie sur la grossièreté provocatrice et jubilatoire du verbe, celui-ci n'en est pas moins remis en question. Aussi le rapport de la littérature au monde apparaît-il problématique : l'obscénité des œuvres souligne l'artificialité du discours littéraire, par le biais du décalage entre fond et forme, tout autant que la fonction créatrice du langage et sa puissance d'évocation. 
À cette première fonction comique peut s'ajouter une fonction édifiante. Il est ainsi possible de voir dans l'évocation obscène du bas corporel un rappel de la petitesse de la condition humaine. La scatologie généralisée d'Audigier, étudié par Chloé Chalumeau, permet de lire l'usage des excréments selon la grille anthropologique du rite d'initiation, ou selon celle, folklorisante, de la renaissance, dans laquelle le cycle de la vie qui se renouvelle est montré par le truchement de la métaphore excrémentielle. Le sexe est aussi ce qui rappelle à l'homme son état de pécheur ; telle est la lecture que suggère Caroline Foscallo du fabliau La Pucele qui voloit voler. Plus largement, Marie-Emmanuelle Simon propose de voir dans l'utilisation du sexe et de la scatologie une argumentation édifiante, facilitant la transmission d'un discours moral qui pourrait être perçu, sans l'apport sulfureux de Luxure, comme austère et insipide. De la même façon, l'exemple de frère Roger, le clerc fornicateur de Guillem de Berguedan, analysé par Pierre Levron, permet de dénoncer les clercs concupiscents, ceux-là mêmes dont les vices sont dénoncés dans les fabliaux.

Il semble par conséquent qu'il n'y ait jamais vraiment d'émancipation morale dans ces textes. Nous pouvons alors nous interroger sur la valeur transgressive de la grivoiserie et de la scatologie. En effet, si la sexualité ainsi présentée paraît réjouissante, elle n’est pas pour autant libérée. Les textes témoignent du respect du discours dominant, ou tout au moins des prescriptions cléricales: même évoquées crûment et explicitement, les positions érotiques par exemple suivent généralement la norme imposée par l'Église. Le surgissement du bas corporel dans les textes n'implique donc pas de transgression morale, pas plus d'ailleurs que de transgression sociale. Le renversement carnavalesque analysé par 
Mikhaïl Bakhtine ${ }^{1}$ semble absent de notre corpus, à l'exception du fabliau Trubert qu'examine Francesco Montorsi. Le monde tel qu'il y est présenté n'est pas bouleversé et les normes sociales y sont respectées. Ce sont généralement les vilains qui se vautrent dans le stupre des fabliaux, ce sont encore eux qui sont arrosés d'excréments. Le cas particulier d'Audigier montre que la fange évoquée dans le texte permet d'opérer avant tout une substitution qui fait tendre l'ensemble vers le burlesque, mais que l'ordre est par ailleurs bien présent et que l'organisation sociale n'y est pas remise en cause. La scatologie relevée dans la lyrique d'oc procède du même mouvement de substitution. La dame idéalisée du discours courtois est remplacée par un être ignoble, sans inversion de la hiérarchie courtoise. Si la présence du bas corporel entraîne une transgression, elle n'est ni morale ni sociale mais peut-être littéraire, remettant parfois en cause les catégories génériques des textes, à l’exemple du problématique Audigier.

La plupart du temps, comme le rappelle Pierre Levron, les textes licencieux ou scatologiques ne critiquent pas un modèle, ils donnent à réfléchir à une autre façon de représenter le monde. Il ne s’agit donc pas de parodies mais bien des «deux faces d'une même production, dont le sens est encore largement à déchiffrer $»^{2}$. En effet, une remarque s’impose pour terminer : elle concerne, comme l'indique Francesco Montorsi, l'absence notable d'études relatives à l'obscénité dans le domaine français, lorsqu'elle est circonscrite à la scatologie ou qu'elle s'étend à un champ plus large alliant sexe et déjections. Une importante part du travail reste donc à mener et les contributions pourraient l'engager vers une lecture qui

${ }^{1}$ Cf. Mikhaïl BAKHTine, L'ÉEuvre de François Rabelais et la culture populaire au Moyen Âge et sous la Renaissance, Paris, Gallimard, 1990 (1 ${ }^{\text {ère }}$ éd. Gallimard 1970/1 ${ }^{\text {ère }}$ éd. russe 1965).

2 Anita Guerreau-Jalabert, «La culture courtoise », in Michel Sot, Jean-Patrice Boudet et Anita Guerreau-Jalabert, Histoire culturelle de la France, 1, Le Moyen Âge, Paris, Seuil, 2005 (1 ${ }^{\text {ère }}$ éd. 1997), p. 207. 
se ferait non pas de façon autonome, mais en regard d'autres textes plus conformes aux normes de la bienséance. C'est bien à la lumière de l'ensemble de la production d'une époque donnée que se joue le sens de ces textes qui deviennent, selon la terminologie empruntée à Pierre $\mathrm{Bec}^{3}$, des « contre-textes », prenant une partie de leur sens dans le contre-modèle qu'ils proposent.

${ }^{3}$ Cf. Pierre BEC, Burlesque et obscénité chez les troubadours : le contre-texte au Moyen Âge, Paris, Stock, « Moyen Âge », 1984. 